\title{
Six types of audio that DEFY reality!
}

\section{A taxonomy of audio augmented reality with examples}

\author{
Michael Krzyzaniak \\ michael.krzyzaniak@yahoo.com \\ RITMO, University of Oslo \\ Oslo, Norway
}

\author{
David Frohlich \\ d.frohlich@surrey.ac.uk \\ DWRC, University of Surrey \\ Guildford, United Kingdom
}

\author{
Philip J.B. Jackson \\ p.jackson@surrey.ac.uk \\ CVSSP, University of Surrey \\ Guildford, United Kingdom
}

\begin{abstract}
In this paper we examine how the term 'Audio Augmented Reality' (AAR) is used in the literature, and how the concept is used in practice. In particular, AAR seems to refer to a variety of closely related concepts. In order to gain a deeper understanding of disparate work surrounding AAR, we present a taxonomy of these concepts and highlight both canonical examples in each category, as well as edge cases that help define the category boundaries.
\end{abstract}

\section{CCS CONCEPTS}

- General and reference $\rightarrow$ Surveys and overviews; General literature; $\bullet$ Human-centered computing $\rightarrow$ Mixed / augmented reality.

\section{KEYWORDS}

audio augmented reality, AAR, augmented reality, AR, audio, music, sound, hearing, listening, enchantment, telepresence, audio overlay, taxonomy, digital mediation

\section{ACM Reference Format:}

Michael Krzyzaniak, David Frohlich, and Philip J.B. Jackson. 2019. Six types of audio that DEFY reality!: A taxonomy of audio augmented reality with examples. In Audio Mostly (AM'19), September 18-20, 2019, Nottingham, United Kingdom. ACM, New York, NY, USA, 8 pages. https://doi.org/10.1145/3356590.3356615

\section{INTRODUCTION}

The concept of Audio Augmented Reality (AAR) has been around for quite some time, as a natural outgrowth of the concept of Augmented Reality (AR) more generally, which

Permission to make digital or hard copies of all or part of this work for personal or classroom use is granted without fee provided that copies are not made or distributed for profit or commercial advantage and that copies bear this notice and the full citation on the first page. Copyrights for components of this work owned by others than the author(s) must be honored. Abstracting with credit is permitted. To copy otherwise, or republish, to post on servers or to redistribute to lists, requires prior specific permission and/or a fee. Request permissions from permissions@acm.org. AM'19, September 18-20, 2019, Nottingham, United Kingdom

(C) 2019 Copyright held by the owner/author(s). Publication rights licensed to ACM.

ACM ISBN 978-1-4503-7297-8/19/09 . \$ \$15.00

https://doi.org/10.1145/3356590.3356615
(1) Enchanting silent physical objects with digital sound

- Enchanted textiles

- Enchanted paper / books / maps

- Enchanted footballs / sports equipment

(2) Deliberate blending of acoustic and digital sound

- Karaoke

- Sound reinforcement

(3) Digital sound-objects placed in real 3d space

- Sound attached to virtual objects in AR Games

- Geolocated sound

(4) Overlay of extra audio information onto the real world

- Conversational Agents

- Self-guided museum tours

- Sat Nav

(5) Realtime digital modification of acoustic sounds

- In-ear translation

- Electric guitar distortion pedals

- Table drums (analysis and resynthesis)

(6) Telepresence - audio merging of remote and local locations

- Telephone

- Treadmill sound walks

- Telematic / networked musical ensembles

Figure 1: List of different types of Audio Augmented Reality

has traditionally given priority to the visual modality. However, various authors writing about AAR all seem to use the term slightly differently, to refer to set of closely related but not identical concepts. Moreover, there have been a great many projects in different fields and spanning a few decades that seem like they qualify as AAR, without haven been conceived with that term in mind; perhaps some of these projects even outdate the term. Of course there already exist definitions and taxonomies of AR but again the focus has been on the visual modality; for example the thoughtful and influential paper at [1], which contains the word 'video' over 70 times in the body of the paper, does not contain 'audio' in the body, and only mentions 'sound' in a few brief and speculative sentences. Often it is possible to arrive at adequate definitions of AAR by isomorphism from video to audio, but because sound and hearing differ in several important ways 
from light and vision, it is worth considering definitions and a taxonomy of AAR separately, in its own right.

We have identified six distinct situations that have been or could be, in our estimation, called AAR. These are listed in Figure 1, accompanied by a few short examples for clarity. Here are a few observations about this list. 1) The categories are not completely mutually exclusive - the 'Enchanted objects' category in particular seems to overlap with nearly everything, yet there are still projects that clearly belong there and not anywhere else, and there are other projects that definitely do not belong there. 2) We not sure that the list is comprehensive, but we don't know of a counterexample at this point. 3) This list takes a user-centered approach. We are not attempting to classify audio hardware, sound reproduction devices, or algorithms; we are classifying experiences, and typically the exact algorithm or loudspeaker arrangement associated with a given experience is interchangeable. 4) The list of examples is not meant to be exhaustive - we are only including the smallest number of the clearest examples to make our point, particularly focusing on archetypes and edge-cases. In each category there are many more people that deserve credit for their incredible work. 5) Throughout this paper we use terms like 'virtual', 'augmented', 'real', and 'synthetic'. Any sensible definitions of these quickly fail under scrutiny, and perhaps some readers will object to our usage in some cases. On the other hand, it is often counterproductive to be overly pedantic about definitions. This taxonomy is intended to be descriptive, not prescriptive, and we hope that suitable definitions of these and related terms will arise through an exhaustive treatment of the concepts, rather than through some pithy criterion.

\section{APPROACH}

We originally devised this taxonomy as part of an industrial design project focused on discovering potential new AAR products and services, and consequently we have adopted a research-through-design approach in assembling this taxonomy. When speculating product ideas, anything can be anything, and ideas quickly morph into a single mega-idea that is both everything and nothing. This taxonomy provided us a conceptual framework that allowed us to guide shapeshifting ideas towards identifiable user needs; when a product idea morphs its way into a separate category, it is useful to consider it a separate product with a different market and fulfilling a different need. Furthermore, we eventually pursued product concepts in each category, which gave us some certainty that we were fully covering the design space. In this sense, this taxonomy has had some real-world validation, insofar as it served as an invaluable foundation to that research.

Having said that, this taxonomy has a few drawbacks worth mentioning. What we have done here is to present a laundry list of things that have been, or could be referred to as AAR. What we have not done is presented an over-arching definition of AAR. Does a system have to be interactive to qualify? Does the inclusion of audio have to be deliberate? Do some of the sounds need to be localized / directional / binaural / immersive? For our research, it was not constructive to draw hard boundaries on these issues, although in another context it might be helpful. For the purpose of this paper, we loosely assume that essential elements of AAR are (1) access to some local physical analogue sound, direct or indirect, and (2) some digitally mediated addition or modification to the sound that provides some benefit. The benefit to the user from the augmentation, or what makes each type of augmentation relevant for the user might be different in different categories, and might include object, activity, place, time, setting, and person, respectively.

In setting out this taxonomy, we have highlighted the independence of the experience from reproduction devices. This notion of format-agnosticism is one of the guiding principles of object-based spatial audio that seeks to represent each component of a sound scene as a distinct object. Within this domain, the recently-proposed approach of device orchestration further loosens the connection between the content and the reproduction by enabling objects to use whatever connected device is most suitable [11][12]. In this context, the challenge of distributing synthetic audio spatially for AAR is shown to be addressable in practical reality for a large number of potential consumers.

\section{ENCHANTING SILENT PHYSICAL OBJECTS WITH DIGITAL SOUND}
Adding meaningful sound to an object that would not otherwise make meaningful sound.
"Audio augmentation refers to an approach which uses auditory feedback to augment users' expe- riences during the interaction with intelligent systems... We embedded playful audio augmen- tation into the [badminton] shuttlecock..." [25]

The concept of enchantment used here comes from David Rose's book Enchanted Objects [30]. Although his focus is not on audio, he discusses at length the idea of using technology to make ordinary objects magical, or to otherwise augment physical objects. Sound is an important medium for enchantment, because it can contain a lot of information in a short time, it can be very responsive, and it does not interfere with many common activities that are visual in nature. This category strongly overlaps with the Overlay of extra audio information onto the real world category, as many enchanted objects are meant to provide some sort of information. Maybe there is a sense in which this category should be a sub-category of that one. On the other hand, a 'magical' 
object provides a much different experience than disembodied information overlay, so we are treating enchanted objects separately. Here are a few examples of items that are very commonly augmented with sound.

\section{Books and Paper}

There are many projects that use conductive ink to turn paper into musical instruments, or to otherwise trigger the playback of sound. Leah Bucheley has been at the center of this work [8][16], and many members of the DIY community have made similar things, such as these musical drawings [31].

David Frohlich has pioneered Audiophotography, and has developed many projects that imbue printed photographs with sound [13]. His more recent work on 'next generation paper' is exploring how audio and other media can be added to each page of a book [14].

\section{Maps}

Sound maps are common enough that they have their own Wikipedia page (s.v. 'Sound map'), and there is a list of nearly 100 of them Brandon Metchley's Dissertation [26]. These projects usually place recordings on a digital map, so users can hear what different locations sound like. The British Library collection is exemplary [23].

Sonic maps are related to sound walks, which we have placed under Telepresence - audio merging of remote and local locations, because to us sound maps seem like they are aimed at augmenting the map itself with audio, whereas sound walks seem like they are more focused on immersing the listener in a remote location, but there is clearly some category overlap here.

\section{Sporting Equipment}

There is an increasing amount of technologically augmented sporting equipment on the market, and because a player's eyes are busy while playing, many of these give the user realtime feedback or coaching cues via sound. The quotation that opened this section is a fantastic example. Systems such as this may encourage people to practice more by making it more fun, and, because sound can be so immediately rewarding, may help guide people towards developing specific skills. Additionally, these systems may be useful as therapies for people with Dyspraxia or other movement disorders. Here is a demo of a football that uses sound to encourage players improve their timing and ball-control [19].

\section{Textiles}

Many people have been interested in knitting, weaving or screen-printing electronic components directly into textiles, for example, by sewing conductive fibres directly into the fabric [37]. This work includes flexible loudspeakers made of steel thread sewn into cloth. One of the authors has a project that involves making drum-pad hats with knitted sensors and cloth loudspeakers [20], so that the hat itself is a complete self-contained digital musical instrument. Other similar items, such as resistive and capacitative textile patches, have been made by others [28] [38].

Somewhat related are musical gloves [41][34][42], hoodies, and other wearables that have sensors embedded in them, that are used as controllers for computer music. These present an interesting edge case - is it the article of clothing that is augmented, or the wearer's body, or something else, or nothing at all? The answer to this certainly varies on a case-by-case basis.

\section{The Human Body}

There have been many projects expressly aimed at turning the human body itself into a musical instrument [40]. This is a clear example of the human body, as an object, enchanted with sound, even if the sound doesn't emanate from the body. Moreover, there have been many interactive or responsive dance works in which a dancer controls synthesized music in realtime using optical motion capture, or sensors attached to the body, for instance [21]. In some cases it might be more correct to say that the entire room or environment has been augmented, rather than the dancer's body.

\section{Arbitrary Objects}

There are a few projects and products that attempt to allow users to imbue arbitrary objects with musical sound, such as Makey Makey [36]. This is essentially a capacitative touch sensor that can be attached to anything, that will relay touch events to a computer via USB HID, which can be, and commonly is, used to trigger sound. Another project aimed at enchanting 'found objects' is in [7], where 'found objects' are items that are not not intended to be musical instruments but are nevertheless used as such. Perhaps tabletop drum kits overlap this category as well, although we have placed them under Realtime digital modification of acoustic sounds because those involve items that make sound on their own, and that sound is intelligently modified or replaced with digital sound. By contrast, this category is for the enchantment of otherwise silent objects.

\section{DELIBERATE BLENDING OF ACOUSTIC AND DIGITAL SOUND}

"CG [computer generated] sound can be displayed using headphones and, using two omnidirectional microphones mounted on the headphone ear-cups, environmental sound can be captured and mixed with the CG sound...We call this audio AR technique 'Microphone-HearThrough' AR” [24]. 
"The user would wear head phones equipped with microphones on the outside. The headphones would add synthetic, directional 3D sound, while the external microphones would detect incoming sounds from the environment" [1].

There are a few devices currently on the market that fit the description in the quotations that opened this section, such as the Roland CS-10EM Binaural Earphones, and the Sennheiser Ambeo Smart Headset. These devices are straightforward analogues of the traditional visual AR headset. However, we would argue that this category is not about the device itself, and in many, if not all cases, this device is unnecessary. It is difficult to think of a plausible, non-contrived situation that necessitates this exact arrangement of hardware. For example, a fully immersive soundscape that mixes acoustic and computer-generated sounds, where all of the sounds are correctly localized in the scene relative to the orientation of the listener's head, can be achieved by placing a loudspeaker in a garden. Additionally, these devices by themselves could be used for any number of applications, including most of the several entries in this taxonomy and many other things besides. The inverse is also true, that any category could be realized on a range of different devices. So it is not the device that is of interest here, it is the concept of seamlessly blending acoustic and digital sound.

\section{Karaoke}

Karaoke is the archetypical example in this category. The acoustic sound of a person's voice is blended with a recording of a band and played through loudspeakers, giving the impression that the person's voice is part of the recording. As an AAR concept, karaoke could perhaps be stronger if it were responsive, for example if the recording followed the tempo and loudness of the singer.

\section{Sound reinforcement}

At concerts and other live audio performances, electronic amplification is used to make the audio louder. In many cases, in particular at classical music concerts, the amplified sound it not meant to completely overpower and replace the live acoustic sound; the sounds are meant to blend together into one unified augmented whole.

\section{Site-specific audio installations}

Part of the reason that garden loudspeakers exist is that there are situations where it is appropriate to listen to recorded audio in a way that does not fully mask the natural soundscape. More generally, site-specific artwork is supposed to interplay with the ambient surroundings of a specific location, and often this involves sound. The work in [15] contains many interesting arrangements. In this work, they put sensors, microphones, loudspeakers, and audio-synthesizing microcomputers in a community garden, and designed several sound generating systems that interact with the local sounds and acoustics of the space itself. The result is a thorough blending of digital and acoustic sounds that places the work in this category. The work also involves amplifying and modifying the sounds of the plants themselves, which overlaps with the Realtime digital modification of acoustic sounds category.

Broadcast radio and background music. These are an interesting example to note since, although long established and almost ubiquitous, they are designed deliberately to augment an otherwise prosaic reality, which reflects the magic, for instance, of a band striking up at a live event. It could be argued that the background music (or muzak) in the retail store as you shop or the hotel lobby as you wait to meet a friend provide an underlay, aimed at soothing your mood. The variety of formats and genres of audio content may offer rich pickings in considering novel AAR experiences: sports commentary, news and documentary, 'reality radio' such as on location interviews, 'slow radio' such as a morning chorus of birdsong, and other podcasts. There is a sense in which this type of radio is more of a 'mixed' reality than an augmented one; the listener might split their attention between the sounds from the radio and those from their real environment, e.g. in the car or in a shop, but the sounds from the radio exist in their own separate virtual world that is unaware of the context in which they are embedded.

\section{OVERLAY OF EXTRA AUDIO DATA OR INFORMATION ONTO THE REAL WORLD}

Using audio to provide a user with additional information that is relevant to their current activity.

"The basic idea [of Audio Augmented Reality] is to superimpose computer generated data on top of the real world, as the person moves within it" [3].

This category is about using sound to provide people with extra context-relevant information. Often this information is triggered by location or proximity. However, this category is distinct from the Digital sound-objects placed in physical $3 d$ space category, because here the sound itself is not treated as a physical object affixed to specific locations in real space, and is not meant to appear to emanate from a definite location.

\section{Navigational aids}

Spoken GPS navigation is perhaps the archetypical example of AAR information overlaid on the real world. Informational sound playback is triggered by your current location and situation. Some readers might initially be confused about 
this classification; location plays a role, it might seem like this is mis-categorized. However, here the sound itself is not affixed to specific locations in real space. This is in sharp contrast to the Digital sound-objects placed in physical $3 d$ space category, which refers to sounds to whose exact location you could point, and which anyone could access given the correct viewport or audioport. Fundamentally, navigational cues are just a personal list of instructions. GPS allows the individual instructions to be delivered just-in-time, which provides extra information overlaid on the task of navigating, but does not place the sounds in $3 \mathrm{~d}$ space, as objects, for anyone to hear in that space. Perhaps to a certain degree the car or dashboard could be seen as an audio enchanted object.

\section{Cooking Coach}

A cooking coach is a hypothetical example that is conceptually identical to navigational aids but that would not rely on location and might therefore be less confusing. A recipe is a list of instructions that could be spoken by speech synthesis. Some system could be devised that detects when the cook completes each step in the recipe, and this would trigger the playback of the subsequent step. The digitally spoken steps are thereby overlaid contextually onto the real activity of cooking.

\section{In-ear museum tours}

Many museums will give you an earpiece to carry around, and when you are in the vicinity of an artwork, you will hear historical information about the work. The quotation that opened this section is a clear example of this category, where the spoken data is about the art and triggered by proximity and potentially the user's browsing history.

In general this is information overlay, as the audio is not generally attached to the artwork. An interesting edge case is the musical instrument museum in Phoenix Arizona, which houses many exotic instruments, and you can hear performances on them through the earpiece. This somewhat blurs the distinction between this and the Digital sound-objects placed in physical $3 d$ space category.

\section{Home appliances}

Your microwave oven (washer-dryer, coffee maker, etc) most likely uses digital sound to give you information about the real world, i.e. it is finished vitiating your food. This seems somewhat like it shouldn't qualify as AAR, but we can't think of a good reason why not. Perhaps because the appliance is really just giving information about the state of the appliance, or perhaps because the sound source can't be taken with you and used to give information about other things.

\section{Chatbots and Conversational Agents}

Chatbots like Siri, Alexa, the Google Assistant, and a surprisingly large number of conversational Japanese fembots can be used to overlay audio information onto the real world, e.g. information about the current weather.

\section{DIGITAL SOUND-OBJECTS PLACED IN PHYSICAL 3D SPACE}

The treatment of digital sound as an ontological token, and placing it at a definite place in the physical world.

"most of the audio AR applications ... use ... tracking (such as GPS) to link the audio content to specific locations"[22].

"Augmented Reality (AR) presentations may be visual or auditory. Auditory presentation ...can ... appear to emanate from specific locations in the user's environment" [39].

This section is about treating audio as if it were a physical object that exists at a specific location, that remains hidden unless revealed with technology. The technology acts as a viewport or audioport that discloses the hidden objects.

\section{Gaming}

The famous game Pokémon Go provides an archetypical example of this type of AR. This game places virtual characters in the players' real environment that can only be seen using the Pokémon app. Some of those characters have sounds attached to them, and by extension, these sounds illustrate this category of AAR. In general, such sounds in an AR game might not be stationary, but are nonetheless intended to be positioned in real $3 \mathrm{~d}$ space as the character moves.

\section{Geolocated Playlists}

Using AAR, people might start leaving their favorite songs or playlists at specific locations. Bluebrain released a geolocated album where each track is placed at a different location in the National Mall in Washington DC, and you can only hear a given track by physically going to the correct location [5]. John Moose has an album that will only play if GPS determines that you are in the woods [4]. In the future, perhaps this will become more common, and the act of listening to the album may be like geocaching or treasure-hunting for the songs.

\section{Art / Museum Projects}

There are a number of public augmented reality art projects that involve sound, where a user has to go to a particular location to hear some audio. Most of these are paired with visual $A R$, and use a smartphone as a viewport or audioport. 
For example, the train station in Kansas City has an app that allows visitors to see and hear real historical people and events overlaid onto the interior if the train station [35].

\section{REALTIME DIGITAL MODIFICATION OF ACOUSTIC SOUNDS}

"We define audio augmented reality as realtime computational mediation of sound..." [18].

\section{Guitar Pedals}

Electric guitars, on their own, tend to have poor sound, and are in some ways the ideal vehicle for all sorts of realtime digital effects processing, filters, reverb, harmonic distortion, delay loops, chorus, phaser, flangers, fuzz, etc. The real sound of the guitar is augmented by computation, and this is the archetypical example in this category.

\section{Augmented acoustic musical instruments}

Within the computer music community, there exists an entire practice of augmenting the natural sound of regular musical instruments with digital effects processing. For example a medieval tromba "augmented with a pickup, speakers and digital signal processing" [2].

Many of these also use a variety of sensors and buttons, such that the instrument can, in addition to being played as a regular instrument, be used as a general-purpose digital control interface, either to control the audio effects parameters, or to control purely synthesized sounds, or to control lighting or other things. Here is an augmented bass clarinet [32]. Here is an augmented saxophone [10]. The practice is common enough that there are metapapers on the topic [27].

In many ways, these might fall in the enchanted objects category, as they are regular objects that have been enchanted with digital technology. However, we have put them here because, in contrast to the other enchanted objects, these objects already make sound, and that sound is modified by technology. The other objects in the enchanted object category do not make meaningful sound to begin with - they are silent objects that are enchanted by virtue of the addition of sound.

\section{In-ear Translation}

The Google translate app now has several AR capabilities. On the visual side, it will overlay a translation of text on real text as seen through a phone's camera. For audio, by analogy, it has a 'conversation' mode that will translate speech to audio in real-time. As a user speaks, their speech will be translated into the target language and re-spoken via speech synthesis. This goes a step beyond guitar pedals and augmented musical instruments insofar as here the entire audio is stripped out and replaced, not just modified with DSP. However, the basic concept here is still the digital mediation of analog sound.

\section{Tabletop Drumkits}

There are several projects that attempt to turn tabletops into drum kits by processing (like a guitar pedal) or replacing (like in-ear translation) the sound of tapping on the table or other object. The most sophisticated of these use machine learning to identify the timbre of each stroke on the table, so that each method of striking the table can be treated differently. A seminal project of this nature is Bruno Zamborlin's Mogees [43], which uses contact microphones placed on a surface to excite digital resonators. Another similar system is described in [33].

As with many of the examples, these might just as well fall under the enchanted objects category, but we have put them here since the idea here is to modify the sound of something that already makes sound, rather than to add sound to something that is otherwise silent. However, this is more of an edge-case as the sound of a tabletop by itself is not that interesting without augmentation.

\section{TELEPRESENCE - MERGING OF REMOTE AND LOCAL LOCATIONS THROUGH SOUND}

\author{
Using sound to merge two or more locations.
}

Traditionally, telepresence is considered a form of virtual, not augmented, reality, insofar as it attempts to give a user the experience of being fully immersed in some remote location. However, there also exist many projects that conceptually merge a user's current location with a remote one, rather than replacing it, which is more augmented in nature. we would argue that when this merging is done via audio, it constitutes AAR.

\section{Telephone (and VoIP / Video Chat)}

There might be people who would argue that telephone is a virtual reality, claiming that the interlocutors occupy a third, virtual space while talking. We would claim that this third space is an augmented merger of the interlocutors real surroundings, because people still interact with their surroundings while talking. For example people drive while talking (and shame on them for it), so clearly they are not fully immersed in some distant virtual space that does not include their real surroundings. For this reason we consider telephone to be a form of AAR.

\section{Sound Walks}

There are many projects that use audio to attempt to give someone the experience of walking around some location other than where they are. Some of these are attached to maps, which makes them related to enchanted objects (enchanted maps), and many are clearly more virtual than augmented. There are also other works that belong firmly in 
this category. Grisha Coleman, Daragh Byrne, David Tinapple, Matthew Mosher, et al, designed a treadmill sound walk [6]. They recorded sound and video of themselves walking or driving around in the desert, and users watch and listen to these recordings while on a treadmill, and the rate of playback depends on the rate of walking. This is clearly augmented and not virtual reality because the act of walking on the treadmill itself is supposed to be part of the experience, in conjunction with the experience of walking through a virtual desert, as both relate to the fictional origin myth that underlies the piece. It is audio AR because it grows out of the tradition of sound walks, and the sound of the desert is used to augment the experience of walking on a treadmill.

\section{Telematic Musical Instruments}

Whereas sound walks typically overlay one remote sound environment on one other local one, telematic musical instruments tend to involve many locations somehow overlaid. In the seminal Telematic Drum Circle [17], many pneumatically controlled drums are in an otherwise empty room, and anyone can play one of the drums remotely by going to a website and tapping on their computer keyboard. In this scenario, one sonic environment is being broadcast out to many people and overlaid on the activity of 'drumming' on a computer keyboard.

In other scenarios, the sonic environment might be overlaid on the activity of playing a real musical instrument; many people might be playing in separate locations, and the sounds are blended together and broadcast to everybody. Normally network latency is an issue that prevents this from working very well, although there is ongoing work to try to deal with that using current network technology [29]. The term 'Internet of Skills' has been recently coined to describe a new interactive paradigm that may emerge once this problem has been trivialized by $5 \mathrm{G}$ networks [9].

Eric Whitacre's virtual youtube choirs overcome the latency by simply not operating in real time. Many people just separately sing one part of a choir piece to a video of a conductor, and later they are mixed together into a composite whole. In this case, we think this is more 'virtual' than 'augmented', in the sense that each singer just sings into a microphone by themselves and that is the end of their involvement. But, it is easy to imagine a low-latency future where this can be done in realtime and each singer can hear all other singers while singing. We think that would be a pretty clear case of telematic AAR.

\section{DISCUSSION}

We have long suspected that there may be a more systematic way of organizing this taxonomy than what we have presented here. All of the examples somehow include something that is in some sense 'real', and something 'synthetic'.

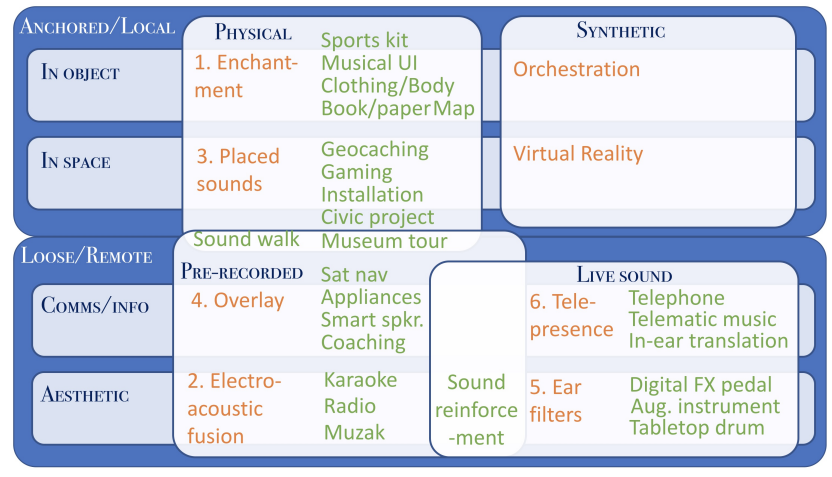

Figure 2: A schematic view of the AAR and VR types (orange) provisionally divided (blue) by the locatedness of overlaid sounds, their pragmatic role, and whether the augmenting elements are synthesised/pre-recorded or physical/live. Examples (green) are grouped with their type, including boundary cases where the characteristics tend to overlap.

It seems like there should be a decision tree - if items $a$, and $b$ are synthetic, while $d$ and $e$ are real, then the work belongs in category $z$. We have not yet found a way of doing this, in part because, as previously mentioned, any sensible definitions of 'real', and 'synthetic' seem to fail under scrutiny, so we are leaving that for future work. Nonetheless, an attempt to summarise these categories graphically is in Figure 2.

\section{ACKNOWLEDGMENTS}

The work presented here was the result of several long discussions with many people. Thanks in particular to Will Buchanan, Russ Bradbury, and Charlotte Edmondson at RPPtv for their boundless creativity and insight. This work was cofunded by RPPtv and Innovate UK.

\section{REFERENCES}

[1] Ronald T Azuma. 1997. A survey of augmented reality. Presence: Teleoperators \& Virtual Environments 6, 4 (1997), 355-385.

[2] Alex Baldwin, Troels Hammer, Edvinas Pechiulis, Peter Williams, Dan Overholt, and Stefania Serafin. 2016. Tromba moderna: A digitally augmented medieval instrument. In Proceedings of the International Conference on New Interfaces for Musical Expression (2220-4806), Vol. 16. 14-19.

[3] Benjamin B. Bederson. 1995. Audio Augmented Reality: A Prototype Automated Tour Guide. In Conference Companion on Human Factors in Computing Systems (CHI '95). ACM, New York, NY, USA, 210-211. https://doi.org/10.1145/223355.223526

[4] Jon Blistein. 2015. Swedish Band Make Album Available Exclusively in the Woods. https://www.rollingstone.com/music/musicnews/swedish-band-make-album-available-exclusively-in-thewoods-189620/ Accessed: 2019-08-07.

[5] Bluebrain. 2011. Announcing 'The National Mall' The First Location Aware Album. https://bluebrainmusic.blogspot.com/2011/03/nationalmall.html Accessed: 2019-08-07.

[6] Grisha Coleman. 2013. Listening as the land talks back: ecology, embodiment and information in the science fictions of echo:: system. 
Leonardo 46, 3 (2013), 204-210.

[7] Stefano Delle Monache, Stefano Papetti, Pietro Polotti, and Davide Rocchesso. 2008. Sonically augmented found objects. (2008).

[8] Stefano Delle Monache, Davide Rocchesso, Jie Qi, Leah Buechley, Amalia De Götzen, and Dario Cestaro. 2012. Paper mechanisms for sonic interaction. In Proceedings of the Sixth International Conference on Tangible, Embedded and Embodied Interaction. ACM, 61-68.

[9] Mischa Dohler, Toktam Mahmoodi, Maria A Lema, Massimo Condoluci, Fragkiskos Sardis, Konstantinos Antonakoglou, and Hamid Aghvami. 2017. Internet of skills, where robotics meets AI, 5G and the Tactile Internet. In 2017 European Conference on Networks and Communications (EuCNC). IEEE, 1-5.

[10] Stuart Favilla, Joanne Cannon, Tony Hicks, Dale Chant, and Paris Favilla. 2008. Gluisax: Bent Leather Band's Augmented Saxophone Project.. In NIME. 366-369.

[11] Jon Francombe, Russell Mason, Philip JB Jackson, Tim Brookes, Richard Hughes, James Woodcock, Andreas Franck, Frank Melchior, and Chris Pike. 2017. Media device orchestration for immersive spatial audio reproduction. In Proceedings of the 12th International Audio Mostly Conference on Augmented and Participatory Sound and Music Experiences. ACM, 33.

[12] Jon Francombe, James Woodcock, Richard J Hughes, Russell Mason, Andreas Franck, Christopher R Pike, Tim Brookes, William J Davies, Philip JB Jackson, Trevor Cox, et al. 2018. Qualitative evaluation of media device orchestration for immersive spatial audio reproduction. Journal of the Audio Engineering Society (2018), 414-429.

[13] David M Frohlich. 2015. Fast design, slow innovation: Audiophotography ten years on. Springer.

[14] David M Frohlich. 2017. From Audio Paper to Next Generation Paper. In Proceedings of the 23rd Brazillian Symposium on Multimedia and the Web. ACM, 9-10.

[15] Lauren Hayes and Julian Stein. 2018. Desert and Sonic Ecosystems: Incorporating Environmental Factors within Site-Responsive Sonic Art. Applied Sciences 8, 1 (2018), 111.

[16] Sam Jacoby and Leah Buechley. 2013. Drawing the electric: storytelling with conductive ink. In Proceedings of the 12th International Conference on Interaction Design and Children. ACM, 265-268.

[17] Byeong Sam Jeon. 2010. Telematic drum circle. Leonardo 43, 1 (2010), 11-11.

[18] Chris Kiefer and Cécile Chevalier. 2018. Towards new modes of collective musical expression through audio augmented reality. In Proceedings of the International Conference on New Interfaces for Musical Expression. Association for Computing Machinery.

[19] Michael Krzyzaniak. 2018. Beethoveen Musical Football Demo. https: //www.youtube.com/watch?v=XyTGSiE1jlA Accessed: 2019-05-20.

[20] Michael Krzyzaniak. 2019. Digital Textiles. https://ambisynth.blogspot. com/2019/01/digital-textiles.html Accessed: 2019-05-20.

[21] Michael Krzyzaniak, Julie Akerly, Matthew Mosher, and Muharrem Yildirim. 2014. Separation: Short Range Repulsion. In Proceedings of the International Conference on New Interfaces for Musical Expression. 303-306.

[22] Tobias Langlotz, Holger Regenbrecht, Stefanie Zollmann, and Dieter Schmalstieg. 2013. Audio Stickies: Visually-guided Spatial Audio Annotations on a Mobile Augmented Reality Platform. In Proceedings of the 25th Australian Computer-Human Interaction Conference: Augmentation, Application, Innovation, Collaboration (OzCHI '13). ACM, New York, NY, USA, 545-554. https://doi.org/10.1145/2541016.2541022

[23] The British Library. [n.d.]. Sound map - Soundscapes. https://sounds. bl.uk/Sound-Maps/Soundscapes Accessed: 2019-05-20.

[24] Robert W Lindeman, Haruo Noma, and Paulo Goncalves de Barros. 2007. Hear-through and mic-through augmented reality: Using bone conduction to display spatialized audio. In Proceedings of the 2007 6th IEEE and ACM International Symposium on Mixed and Augmented Reality. IEEE Computer Society, 1-4.

[25] Yudan Ma, Tilde Bekker, Xipei Ren, Jun Hu, and Steven Vos. 2018. Effects of Playful Audio Augmentation on Teenagers' Motivations in Cooperative Physical Play. In Proceedings of the 17th ACM Conference on Interaction Design and Children (IDC '18). ACM, New York, NY, USA, 43-54. https://doi.org/10.1145/3202185.3202729

[26] Brandon Michael Mechtley. 2013. Techniques for Soundscape retrieval and synthesis. Arizona State University.

[27] Dan Newton and Mark T Marshall. 2011. Examining How Musicians Create Augmented Musical Instruments.. In NIME. 155-160.

[28] Ivan Poupyrev, Nan-Wei Gong, Shiho Fukuhara, Mustafa Emre Karagozler, Carsten Schwesig, and Karen E Robinson. 2016. Project Jacquard: interactive digital textiles at scale. In Proceedings of the 2016 CHI Conference on Human Factors in Computing Systems. ACM, 4216-4227.

[29] Michael Rofe and Federico Reuben. 2017. Telematic performance and the challenge of latency. Fournal of Music, Technology \& Education 10, 2-3 (2017), 167-183.

[30] David Rose. 2014. Enchanted objects: Design, human desire, and the Internet of things. Simon and Schuster.

[31] Eric Rosenbaum. 2012. MaKey MaKey Musical Paintings. https: //www.youtube.com/watch?v=uqPys4opLn8 Accessed: 2019-05-20.

[32] Sébastien Schiesser and Jan C Schacher. 2012. SABRe: The Augmented Bass Clarinet.. In NIME.

[33] Diemo Schwarz, Grégoire Lorieux, Emmanuelle Lizère, Ariadna Tarrès, and Frédéric Bevilacqua. 2017. A Topo-Phonic Table for Tangible Sonic Interaction. In International Computer Music Conference (ICMC).

[34] Stefania Serafin, Stefano Trento, Francesco Grani, Hannah PernerWilson, Sebastian Madgwick, and Thomas J Mitchell. 2014. Controlling physically based virtual musical instruments using the gloves. (2014).

[35] Samantha Shankman. 2014. U.S. Train Station Portrays History in Augmented Reality App. https://skift.com/2014/11/21/u-s-trainstation-portrays-history-in-augmented-reality-app/ Accessed: 201905-22.

[36] Jay Silver, Eric Rosenbaum, and David Shaw. 2012. Makey Makey: improvising tangible and nature-based user interfaces. In Proceedings of the sixth international conference on tangible, embedded and embodied interaction. ACM, 367-370.

[37] Matteo Stoppa and Alessandro Chiolerio. 2014. Wearable electronics and smart textiles: a critical review. sensors 14, 7 (2014), 11957-11992.

[38] Paul Strohmeier, Jarrod Knibbe, Sebastian Boring, and Kasper Hornbæk. 2018. zPatch: Hybrid resistive/capacitive etextile input. In Proceedings of the Twelfth International Conference on Tangible, Embedded, and Embodied Interaction. ACM, 188-198.

[39] V. Sundareswaran, Kenneth Wang, Steven Chen, Reinhold Behringer, Joshua McGee, Clement Tam, and Pavel Zahorik. 2003. 3D Audio Augmented Reality: Implementation and Experiments. In Proceedings of the 2Nd IEEE/ACM International Symposium on Mixed and Augmented Reality (ISMAR '03). IEEE Computer Society, Washington, DC, USA, 296-. http://dl.acm.org/citation.cfm?id=946248.946841

[40] Atau Tanaka and Marco Donnarumma. 2018. The body as musical instrument. In The Oxford Handbook of Music and the Body.

[41] Seth Dominicus Thorn. 2018. Alto. glove: New Techniques for Augmented Violin. In Proceedings of the International Conference on New Interfaces for Musical Expression. Blacksburg.

[42] Giuseppe Torre, Kristina Andersen, and Frank Baldé. 2016. The Hands: The making of a digital musical instrument. Computer Music fournal 40, 2 (2016), 22-34.

[43] Bruno Zamborlin. 2015. Studies on customisation-driven digital music instruments. Ph.D. Dissertation. Goldsmiths College (University of London). 